\title{
Dealing with the Avalanche of Data Generated in High Data Rate Macromolecular \\ Crystallography
}

Jean Jakoncic ${ }^{1}$, Herbert J. Bernstein ${ }^{2}$, Alexei Soares ${ }^{1}$, Wuxian Shi ${ }^{3}$, Martin Fuchs ${ }^{1}$, Robert Petkus ${ }^{1}$, Robert M. Sweet ${ }^{1}$, Sean McSweeney ${ }^{1}$

${ }^{1}$ Brookhaven National Laboratory, Upton, NY, USA;

${ }^{2}$ Rochester Institute of Technology, Rochester, NY, USA;

${ }^{3}$ Case Western Reserve University, Cleveland, Ohio;

Newly commissioned state of the art $M X$ beamlines fitted with current advanced hybrid pixel detectors are now in operation. At the NSLS-II, AMX and FMX, two high-brightness microfocusing beamlines ( $>10^{11}$ and $>5 \times 10^{12} \mathrm{ph} / \mathrm{s} / \mathrm{um}^{2}$ respectively) are fitted with Dectris Eiger detectors and are equipped with advanced automation that will ultimately allow screening of up to 1000 crystals per day. We have seen throughput greater than $1 \mathrm{~GB} / \mathrm{s}$ per beamline during demanding experiments and are expecting this to increase in the upcoming months. With this level of throughput, near real time data analysis feedback is a necessity. This requires infrastructure with a high bandwidth network, fast-I/O large storage and significant computational capacity. Optimized data processing software and pipelines are being developed to help in coping with the throughput. We will present the state of current problems that the community is facing and some of the solutions that are currently deployed at various facilities.

This will be followed by two talks by Intel and a facilitated discussion. The first talk is on

$$
\text { Intel Scalable System Framework (HPC) }
$$

The second talk is on

Intel software and tools Eco-system (HPC)

and will focus on adapting MX code to use Intel processors effectively. 